\title{
Dewatering to higher densities — an industry review
}

\author{
F. Schoenbrunn FLSmidth Inc., United States of America
}

\begin{abstract}
The development of high density and paste thickening technologies has changed the economics used in evaluating the benefits of increased thickener underflow density. This comes at a time of increasing competition for scarce water resources and a tightening regulatory framework in both water use and impoundment. These and other factors have led to more and more plants using high density and paste thickening technologies together with optimised tailings disposal schemes to recover water, minimise tailings impoundment areas and comply with regulations. High density and paste thickening have allowed these plants to take advantage of the tailings disposal options that weren't previously possible. This paper will give an overview of technology including the differences between high rate, high density and deep cone paste thickeners. It will also review a number of recent projects including the decision process and resulting technology selection. Results from recent installations will also be discussed.
\end{abstract}

\section{Introduction}

Maximising thickener underflow density has advantages in many of the traditional thickener applications. There is a long history of innovations in thickener design to enable the production of higher densities. Large High Density thickeners were pioneered in the late 1980s. The roots of Deep Cone thickeners go back to the 1960s. Over the last two decades significant advances in the design of thickeners has enabled production of higher densities than was traditionally possible. This started with smaller tonnage machines but now extends to all production sizes.

The technology has significant benefits and has been proven in applications such as leach feed, CCD circuits, underground backfill, filter feed and tailings disposal.

Various manufacturers use different terminology for these designs. Many of them segregate the designs by the rheology of the underflow. FLSmidth uses the terms High Density and Deep Cone, Westech uses the terms High Density and Deep Bed, Delkor uses High Density and Paste, and Outotec uses the terms High Compression and Paste. This paper uses the terms High Density Thickener (HDT) and Deep Cone Thickener (DCT). In general these are all designed to produce underflow rheologies above what can be achieved in High Rate or Conventional thickeners, which are typically limited to about 20 or 30 Pascal yield stress. High Density thickeners are generally designed for the range of 30-100 Pa, and Deep Cone, Deep Bed or Paste thickeners for over $100 \mathrm{~Pa}$. It should be noted that these designs cover a wide range. The difference between a thickener designed for $30 \mathrm{~Pa}$ versus one designed to produce a $100 \mathrm{~Pa}$ underflow is very significant and has a large impact on the cost.

In relation to thickener performance, underflow rheology is typically discussed in terms of its yield stress. At low solids concentrations slurries behave as essentially Newtonian fluids. However, at a high enough concentration, the particle interactions increase to a point where the slurry exhibits a resistance to deformation. The yield stress is a function of the solids concentration and typically increases rapidly as the solids concentration approaches that of a fully connected matrix. This is a fairly complex subject, but it should be noted that most mineral slurries that are concentrated enough to exhibit a yield stress are also subject to a phenomena called shear thinning, where imparting some energy into the slurry reduces the yield stress. It is typical for a sheared yield stress to be $40 \%$ or more lower than the corresponding unsheared yield stress taken at the same solids concentration. The material inside a thickener is generally unsheared and the thickener design needs to be capable of handling and transporting this material internally. Some thickener manufacturers have designs to take advantage of this phenomena. 


\section{Development of thickening technology}

Thickeners were invented in the early 1900s though batch settling tanks go back much further. John Dorr patented the continuous thickener in 1906, which included a circular tank and a set of rakes. Starches and guar were used as coagulants to agglomerate the solids and improve settling rate and overflow clarity. In the 1960s, starches and guar were supplanted by synthetic flocculants which were dramatically more effective. The use of flocculants is now standard on most thickeners with the exception of processes that can't tolerate contamination. The use of flocculants brought out the need for feed dilution to optimise the effect. Feed dilution dates back to the 1950s, when Harry Cross patented a windows style feedwell dilution system. High Rate thickeners were pioneered in the sugar industry by Enviroclear in the 1980s and operated with a deep feedwell and floc injection into the bed.

Developments in thickening technology over the past 15 years have enabled significantly higher underflow densities to be consistently produced. This technology started as an extension of high rate thickening, utilising a deeper mud bed to augment the thickening capacity. High density or high compression thickeners usually add depth to a high rate design to aid in increasing the underflow density. Deeper mud beds increase the mud compressive force, reducing the time required for thickening and increasing the underflow density. There are a number of modifications necessary to be able to produce and handle the higher viscosity materials, including significantly higher torque capacity than high rate machines, low drag rake arms, pickets, higher raking capacity and improved underflow discharge.

Deep cone thickeners (DCT) are a newer alternative which are designed around production of high viscosity muds. They utilise very deep mud beds and steep floors to be able to produce and discharge muds near the limit of pumpability and operate at very high rates in terms of thickener area. Currently these designs are being built up to about $45 \mathrm{~m}$ diameter although, as is typical in the minerals industry, larger machines are on the drawing board.

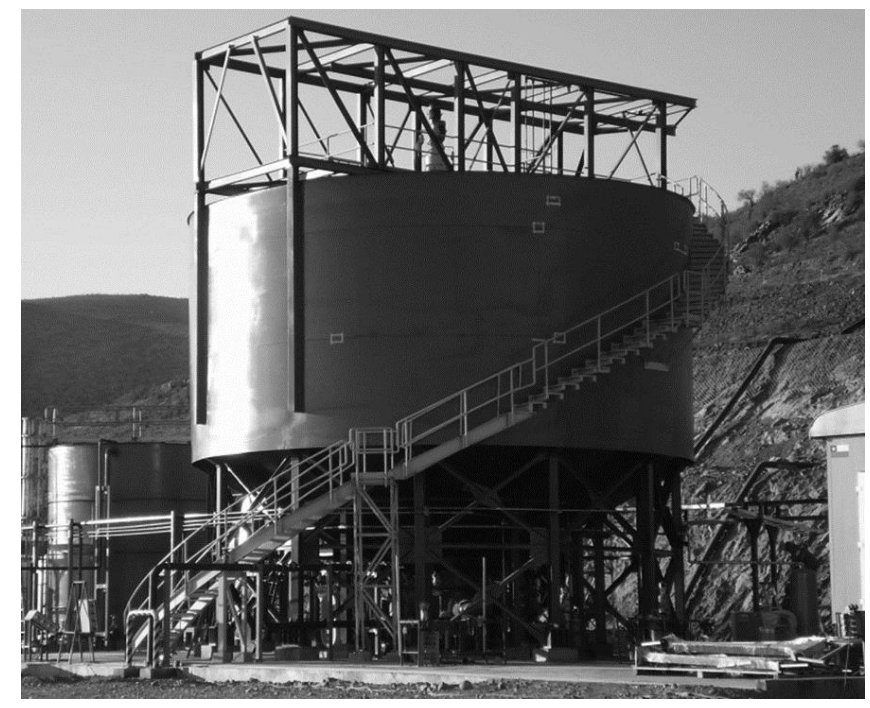

Figure 1 Typical deep cone thickener

In some applications, underflow with the consistency of paste can be achieved by high rate, high rate rakeless, or high density machines. However, deep cone thickeners are currently the best technology for achieving maximum underflow densities utilising sedimentation equipment alone. These units typically utilise very deep mud beds in order to take maximum advantage of mud compressive forces for dewatering and provide sufficient time for the mud to dewater to a paste consistency. The tank height to diameter ratio is frequently 1:1 or higher. Due to the high underflow viscosities, mechanism torques can be 5-10 times higher than high rate machines on similar materials in order to be able to handle performance excursions where very high viscosity material is produced.

In the 1960s and 1970s the British National Coal Board (NCB) developed and installed a number of Deep Cone Thickeners (DCTs) to process coal flotation refuse. They were designed to take advantage of modern 
flocculants that were newly developed at that time. These early designs utilised a steep bottom cone and a rotating picket assembly to produce underflow solids concentrations high enough to be discharge onto a conveyor belt. There were operational difficulties, but for a first cut, these units operated remarkably well.

Alcan built on the technology for red mud washers, but pumped the underflow and used dry tailings disposal methods (Robinsky, 1978) with the thick mud. These were used in the red mud washing circuits and markedly improved the CCD circuit efficiency. Alumina remains the market with the highest level of penetration for the technology.

In the mid 1990s the technology expanded outside of the coal and alumina industries. Eimco Process Equipment licensed the DCT technology from Alcan at that time and began to market it for other markets in the minerals field. Around the same time, Inco and Dorr Oliver developed the PPSM, which uses some of the same principles and is also designed to produce paste type discharge rheology.

In the late 1980s and early 1990s, work had been done to improve thickener performance by operating with a deeper bed. These high density units designed and built by both Outokumpo and Eimco were generally designed with higher torque and 2 or $3 \mathrm{~m}$ of bed depth compared to a typical $1 \mathrm{~m}$ bed depth, but usually with a conventional 1:6 floor slope. They typically produce higher underflow concentrations than achievable with standard thickener designs, but a bit lower than achievable with a DCT. These designs had some notable successes but market penetration was slow. Once DCTs were introduced and proven, the market shifted and began to accept and roll out that technology in a more widespread fashion. Even though the DCT technology is well proven and accepted, the HDT range of designs is generally more favoured depending on application and requirements.

There are now several hundred installed DCTs and the technology continues to be developed. There is over 30 years of development of Alcan Deep Thickener Technology in the alumina industry and 15 years in applications outside the alumina industry. The list of applications and markets is growing rapidly as operators move to take advantage of the technology.

Over the last 10 years engineering firms have come to accept and begun looking at using the new capabilities. The shift in thinking has been going on for a while but has only recently begun to see widespread implementation (Schoenbrunn, 2005). In the last five years it has become commonplace for new projects to incorporate higher tailings densities in their design. Engineering for a new plant now normally at least considers high density or paste thickening. Where design underflow densities for a copper tailings in the 1990 s were typically $50-60 \mathrm{wt} \%$, today most new plants are being designed to produce $60-70 \mathrm{wt} \%$ underflow.

Specifications for new thickeners have recently started to include terms such as 'high density' or 'paste'. As noted above, this does little to specify the intended duty other than to indicate that the customer would like better performance than could be obtained with a standard high rate thickener. It is preferable for a target rheology to be nominated. In any case, if a client intends to buy on low price and uses a term such as high density, they should be aware that different manufacturers will have different interpretations for what that might mean.

\section{Principles}

The production of a thickener underflow of a maximum density can be achieved with practically any gravity sedimentation device, given enough area, settling time, and raking capability. The differences noted with the DCT are in the design details which are directed towards achieving this limiting concentration at a very high loading in terms of solids throughput per unit area. Five factors enter into this picture - the optimisation of flocculation procedures:

- Relatively deep pulp depth.

- Relatively steep floor slope.

- High torque and raking capacity of the mechanism; torque requirements are a function of the yield stress.

- The use of mechanical devices, such as rakes and pickets, to 'work' the compacting slurry. 
- Sufficient mud retention time.

Good flocculation is a key to the successful operation of almost any thickener and careful attention needs to be paid to this aspect of the thickener design. Optimum flocculation requires selecting the best reagent for the particular tailings and the introduction of the reagent at an optimum slurry concentration in order to promote better flocculation. Generally, this concentration is lower than the normal process concentration and dilution of the slurry will be necessary for minimum flocculant use and maximum solids settling rate. There are several designs for self-diluting feedwells, where the liquor within the thickener is recycled to the feedwell to optimise the flocculation step. The self-diluting feedwell design is frequently the single most critical aspect of the thickener in terms of improved performance. Considerable work is being done to optimise these designs, typically involving computational fluid dynamics modelling. Two notable designs are the FLSmidth E-Volute variation of the E-Duc self-diluting feedwell and the Outotec vane feedwell.

In the case of HDTs and DCTs, the sizing procedures generally must take into account the volume of thick pulp within the thickener, as a substantially longer than normal retention time is necessary for the solids to obtain high concentrations. It is generally more economical to provide this volume by using a relatively deep bed of pulp rather than a greater area with a shallow compaction bed. This increase in depth also provides bed compression, where the weight of solids above helps compress and dewater the mud to higher concentrations.

The added depth results in greater loads being imposed on the raking mechanism, particularly since the thick mud will be found even at the perimeter of the thickener. This results in a substantial increase in load on the drive and it is necessary to use as much as an order of magnitude more torque for the drive mechanism as would be used on a similarly sized thickener producing a low yield stress underflow concentration. In addition, the design criteria for the drive are significantly different from normal minerals duty. The torque will be consistently high, but will not be subject to as many peaks. Due to the high pulp depth, there is not much to be gained by including a rake lifting mechanism, since the pulp depth is typically much higher than the rake lift height.

As the thickened mud approaches a limiting concentration, it behaves less and less like a fluid, and has little tendency to flow to the underflow withdrawal point. Therefore, steep floors of 30-60 degrees and rakes designed to overcome the yield stress of the mud are used to transport the thickened mud to the outlet. By contrast, standard thickener design calls for a slope of something less than 10 degrees.

When the pulp reaches the compression zone, mechanical action, such as by the raking mechanism itself, contributes to the rate of water removal from the compacting mass. Since DCTs operate with pulp depths which generally extend well above the rake structure, the mass of material located in the zone is not exposed to a similar mechanical action. Therefore, it is helpful to add pickets, usually consisting of posts or rods which project into this mass, in order to create channels and assist in water removal. The use of low drag designs is important to minimise the torque required.

Underflow recycle is used on many Alcan style DCTs to shear-thin the underflow. The thinned underflow (reduced yield stress) is reinjected into the underflow cylinder to aid in removing unthinned mud. The thinned mud acts as a carrier fluid and helps eliminate problems between the underflow cylinder and the centrifugal pumps. The Alcan shear thinning loop is patented. At least one variation on this has been developed and patented.

\section{$4 \quad$ Applications}

A number of new copper plants are utilising high density tailings thickeners. These improve water recovery and minimise tailings impoundment requirements. High density thickeners can typically achieve 5-10 wt\% higher underflow densities compared with a high rate thickener, achieving densities that can still be moved with centrifugal pumps and pumped to the tailings impoundment without positive displacement pumps. This increase in underflow density helps minimise water consumption, particularly if the density is high enough to minimise free water discharge (Vietti et al., 2010). High density and deep cone thickener technology is having a significant impact on plant design and operation. A few examples of copper concentrators taking advantage of the technology in the past three or four years are listed here.

- Sar Cheshmeh, Iran, (12) 24 m DCTs, processing 96,000 tpd 60-62 wt\% underflow. 
- Wu Shan, China, (2) 40 M DCTs, 35,000 tpd, 65 wt $\%$.

- Toromocho, Peru, (3) 43 m High Compression, 124,000 tpd, 70 wt $\%$.

- Andina, Chile, 43 m HDT, 25,000 tpd, 68 wt $\%$.

- Esperanza, Chile, (3) 60 m HDT, 110,000 tpd, 67 wt\%.

- Coemin, Chile, 22 m DCT, 7,700 tpd, 68 wt\%.

- Caserones, Chile, (3) 45 m HDT, 50,000 tpd, 62 wt $\%$.

- Rosemont, USA (2) $50 \mathrm{~m}$ HDT, 67,000 tpd, $65 \mathrm{wt} \%$ followed by pressure filtration for dry stacking.

- Worldwide there are roughly 200 DCTs installed and operating.

In side by side pilot testing with multiple manufacturers, large differences in performance have been seen which suggests that differences in the equipment and operation can have a large impact.

\section{Conclusions}

Care should be taken in specifying the desired performance for a thickener. Defining the expected operating yield stress range in addition to the underflow density gives the thickener designer the information needed to optimise the thickener for the intended duty.

Thickening to higher underflow densities using High Density and Deep Cone thickening technologies is rapidly becoming widespread practice. A substantial increase in density is being achieved in a variety of plants across a range of industries and applications.

Engineers, operators, plants and companies cannot afford to miss the opportunities afforded by high density and deep cone thickeners. Reduced capital and operating costs, improved recoveries, and reduced environmental impact have made these technologies a permanent part of the plant design toolbox.

\section{References}

Robinsky, E.I. (1978) Tailings Disposal by the Thickened Discharge Method for Improved Economy and Environmental Control, Proceedings of the Second International Tailing Symposium, Denver, U.S.A.

Schoenbrunn, F. (2005) High Tonnage Surface Stacking; Impediments to Implementation, in Proceedings Eighth International Seminar on Paste and Thickened Tailings (Paste05), R.J. Jewell and S. Barrera (eds), 20-22 April 2005, Santiago, Chile, Australian Centre for Geomechanics, Perth, pp. 251-259.

Vietti, A.J., Boshoff, J.C.J. and Cope, A. (2010) Does Thickening Save Water? the 4th International Platinum Conference, Platinum in Transition 'Boom or Bust', The South African Institute of Mining and Metallurgy. 
